\title{
Multi-level Evaluation of Load Supply Adequacy for Transmission Network
}

\author{
Zheng-lin Yang, jian-guo Yao, jian Geng \\ China Electric Power Research Institute, Nanjing, China \\ Email: yangzhenglin@epri.sgcc.com.cn, yaojianguo@epri.sgcc.com.cn, gengjian@epri.sgcc.com.cn
}

Received March, 2013

\begin{abstract}
Considering sharp increase of central integration scale of intermittent energy generation, power system couldn't rely on the determined model based operation schedule to assure its reliable load supply. Then, this article presents a new load supply adequacy evaluation method, which based on continuous security constrained generation optimization, and provides the functional architecture design of online multi-level short term load supply adequacy evaluation system.
\end{abstract}

Keywords: Load Supply Adequacy; Operation Schedule; Security Analysis; Continuous Security Constrained Generation Optimization; Unit Commitment; Economic Dispatch

\section{Introduction}

It is the main objective of short term operation schedule to secure power system operation, improve load supply reliability and optimize generation resource configuration. Outage schedule, one type of operation schedule, should provide enough margins for reliable power system operation by optimizing transmission network topology. Generation schedule, another type of operation schedule, should optimize multi-periods unit commitment status and output according to predefined objective, taking consideration of power supply balance, transmission capability constraints and generation operation constraints. In traditional deterministic model for short term operation schedule optimization, outage schedule and generation schedule are optimized on the assumption that all generation units meet certain reliability level in operation, and the negative influence of load forecast bias and forced outage of transmission and generation device could be avoided by scheduled regulation reserve and spinning reserve.

With integration of large scale intermittent energy generation, such as hydropower, wind power and photovoltaic power, its negative influence on operation has far exceeded load forecast bias. The traditional generation schedule optimization model, which treat intermittent energy power as fixed generation and eliminate it from system load according to forecast of hydropower, wind power and photovoltaic power, could not cope with the random fluctuation introduced by large scale intermittent energy generation. It is important to arrange enough margins in outage schedule optimization to meet addi- tional reserve requirement caused by intermittent energy generation. Therefore, it is of great value for improving load supply reliability to accurately evaluate maximized load supply capability for transmission network. Paper [1] presented load supply adequacy index for bus, which is defined as the ratio of maximized load supply capability to current bus load, and applied in load supply capability evaluation of buses. It is the main objective of outage schedule optimization and evaluation to arrange enough available generation capacity and transmission capacity for coming generation optimization in order to cope with fluctuation of load. This article applies load supply adequacy index in multi-level evaluation of load supply capability on every bus according to outage schedule. Load supply adequacy evaluation could provide quantitative margin information to assist operator evaluating the adaptability of power system for load fluctuation. Through the evaluation of load supply adequacy index, the transmission network planner and operator could evaluate the quantitative security margin of transmission network on the basis of qualitative security [2] evaluation of operation schedule, and also could evaluate the adaptability for integration of intermittent energy generation.

In the reference documents, load supply adequacy index of every bus is evaluated by increasing load continually under the assumption that all other bus load remaining unchanged. The unreasonable assumption restricts its application in engineering. Firstly, there is strong relationship between bus loads variation. Secondly, it is too conservative to evaluate load supply adequacy index by increasing generation at equivalent ratio to meet bus load growth. Hence, this article presents multi-level load sup- 
ply adequacy evaluation method, which evaluates load supply adequacy from bus, zone and system level, and integrates sensitivity factor based evaluation method and continuous security constrained generation optimization based evaluation method[3-8] to make the index more accurate reflect load supply adequacy of transmission network. Based on multi-level load supply adequacy evaluation, the online short term load supply adequacy evaluation system is designed and implemented.

\section{Theory of Load Supply Adequacy Evaluation}

\subsection{Sensitivity Factor Based Evaluation Method}

Paper 1 presents bus load supply adequacy index and sensitivity factor based evaluation method for the first time. The theory is as follows.

First of all, sens $\mathrm{ij}_{\mathrm{ij}}$, the sensitivity of load $\mathrm{j}$ on branch $\mathrm{i}$, is calculated. Then, the maximized load supply capability $\mathrm{P}_{i j \text {, } \max }$ on bus $\mathrm{j}$ is calculated considering available transmission capability of branch $i$ by increasing generation output by equivalent ratio and assuring balance between generation and load. Considering all branch, the minimized

$$
P_{j, \text { max }}=\min \left\{P_{1 j, \text { max }}, P_{2 j, \text { max }} \cdots P_{i j, \text { max }} \cdots\right\}
$$

is defined as load supply capability of bus $\mathrm{j}$ and $\eta_{\mathrm{j}}$ is defined as load supply adequacy of bus $j$.

$$
\begin{gathered}
\frac{\mathrm{f}_{\mathrm{i}, \max }-\mathrm{f}_{\mathrm{i}}}{\text { sens }_{\mathrm{ij}}}=\mathrm{P}_{\mathrm{ij}, \max }-\mathrm{P}_{\mathrm{j}} \\
\eta_{\mathrm{j}}=\frac{\mathrm{P}_{\mathrm{j}, \max }}{\mathrm{P}_{\mathrm{j}}}
\end{gathered}
$$

$f_{i, \text { max }}$ represents thermal limit of branch $i, f_{i}$ represents the power flow of branch $\mathrm{i}$ at initial state, $\mathrm{P}_{\mathrm{ij}, \max }$ represents the maximized available load supply capability of bus load $\mathrm{j}$ considering the transmission constraint of branch i.

sens $_{\mathrm{ij}}$ Indicates the relationship between branch $\mathrm{i}$ and load $\mathrm{j}$, larger sens $_{\mathrm{ij}}$ represents stronger relationship between them. The difference between $\mathrm{f}_{i, \max }$ and $f_{j}$ represents the available transmission capability of branch i considering its thermal limit. The smaller difference between $\mathrm{f}_{i \text {, max }}$ and $f_{j}$, indicates the smaller load supply adequacy $\eta_{\mathrm{j}}$ of load $\mathrm{j}$, which has strong relationship with branch i.

Considering thermal limit of branch and its relationship between bus load, sensitivity factor based evaluation method evaluates the load supply adequacy by selecting the minimized load supply capability among all the maximized load supply capability considering thermal limit constraints of all branches. In sensitivity factor based evaluation process, not only thermal limit of branch, but also the transient limit of interface and limitation of generation capability has great influence on load supply adequacy of bus load.

Sensitivity factor based evaluation method has two shortcomings in engineering application. Firstly, it is only suitable for single bus load supply adequacy evaluation. Secondly, it is more conservative since it rely on proportional generation growth other than consider more rational generation output and unit commitment.

\subsection{Continuous Security Constrained Generation Optimization Based Evaluation Method}

The theory of continuous security constrained generation optimization based method is to calculate the maximized load supply capability through continuous load growth and corresponding generation optimization to find feasible generation schedule, which could satisfy all the power balance constraints, transmission security constraints and generation security operation constraints. The evaluation process of continuous security constrained generation optimization based method is demonstrated as Figure 1.

Ld0 represents initial load of evaluation object, and the growth ratio of each round is $\delta$. The first round evaluation starts after the setting of objective and constraint model for generation optimization. In every round, base

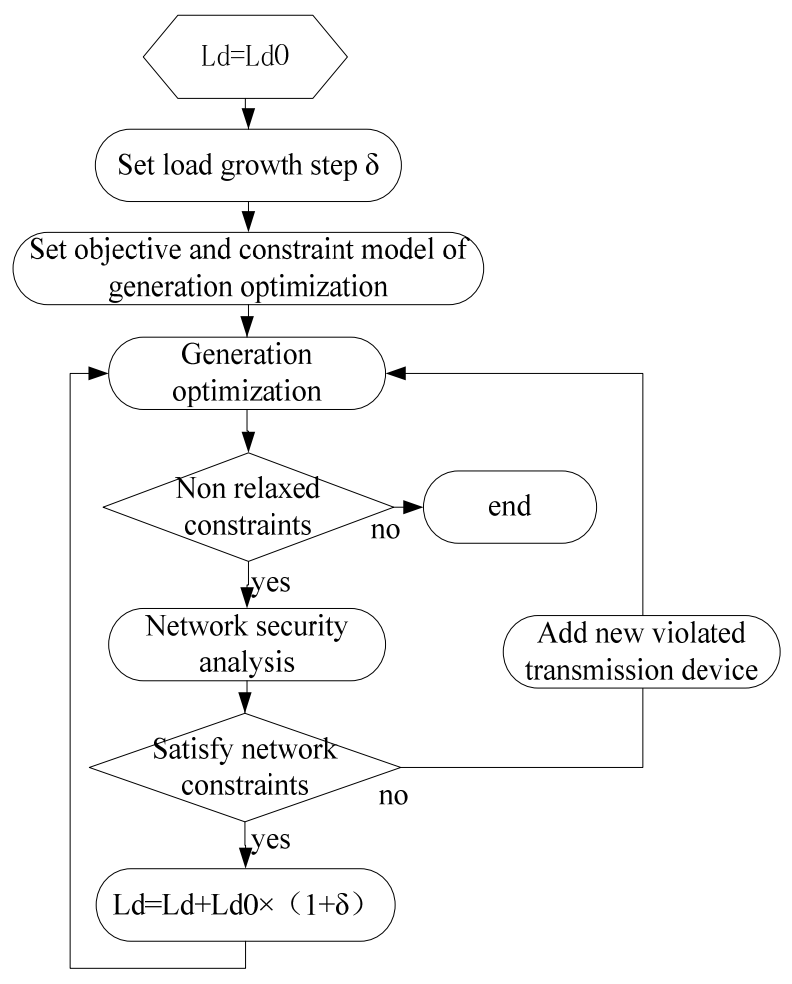

Figure 1. Diagram of continuous security constrained generation optimization based evaluation method. 
case security analysis and contingency analysis should be carried out on achieved feasible generation schedule after generation optimization. And if any new violated transmission devices are detected, the new violations should be sent to generation optimization engine as additional transmission security constraints until no other violations appear. Then increase the load by \& and move to next round evaluation. On the other hand, if generation optimization engine could not find feasible generation schedule that satisfy all constraints, the constraints are relaxed and the ld of last round should be taken as the maximized load supply capability, and $L d / \operatorname{ld} 0$ should be load supply adequacy of evaluation object. Then the evaluation process ends. Since evaluation load grows and security constrained generation optimization run every round, it is called continuous security constrained generation optimization based evaluation method.

In generation optimization model, the constraints can be divided into system power balance, generation operation and transmission security. For unit commitment, the constraints include system power balance, minimized regulation reserve, maximized unit output, minimized unit output, ramp up and ramp down speed, initial unit status, minimized run time, minimized offline time, unit regulation reserve, must run, fixed unit commitment or output schedule for generation, transmission capability in base case and contingency analysis, and also include the maximized output, minimized out, maximized energy, minimized energy, minimized regulation reserve and spinning reserve for a group of generations.

Any generation schedule, which satisfy all the constraints listed above for certain load level, is feasible for load supply adequacy evaluation, which has no specific requirement on optimization objective. According to available cost model of generation, the optimization objective could be one of minimized energy cost, minimized fuel cost or minimized bias of yearly energy contract accomplishment between generation providers. Maximized transmission margin could also be taken as optimization objective by converting from load ratio of transmission device into virtual dispatch cost. Maximized transmission margin optimization could achieve the best performance with moving of transmission capability constraints to optimization objective model.

Compared with sensitivity factor based evaluation method, continuous security constrained generation optimization based evaluation method not only could be used for single period load supply adequacy evaluation, but also could be used for accurate multi-period coupled evaluation, and the evaluation result could be more reasonable. For single period evaluation, the ramp speed of generation need not be considered in generation optimization. But for multi-period evaluation, all the constraints listed above should be taken into consideration for con- tinuous load growth. Considering high strength optimization, the calculation of continuous security constrained generation optimization based evaluation method need more time than sensitivity factor based evaluation method.

\section{Multi-level Load Supply Adequacy Eval- uation of Transmission Network}

\subsection{Level of Short Term Load Supply Adequacy Evaluation}

The level of short term load supply adequacy evaluation could be divided into bus, zone and system. Bus level evaluation takes single bus load as evaluation object, calculates maximized load supply capability of bus considering continuous load growth and assumption of any other bus load remaining unchanged. For the strength relationship between bus loads, the situation that one bus load grows continuously and all other remain unchanged seldom appears. The result of bus load level evaluation could not reflect load supply adequacy of transmission network intuitively and is inconvenient to apply in engineering. Power system operator care more about the load growth margin of a zone or even all the system than bus level. Power system operator not only care load supply adequacy of one period, but also care the load supply adequacy of all periods of a day to evaluate the maximized margin of load supply for random fluctuation of all kinds such as cased by intermittent energy resource.

Zone level evaluation takes single zone as evaluation object, calculates maximized load supply capability of zone considering continuous load growth and assumption of any other zone load remaining unchanged. According to evaluation time scope, zone level evaluation could be divided into single period and multi-periods evaluation.

System level evaluation takes all the system as evaluation object, calculates maximized load supply capability of system considering continuous system load growth and assumption of tie line exchange remaining unchanged. System level evaluation also could be divided into single period and multi-periods evaluation.

\subsection{Load Growth Model}

In order to reduce the evaluation times, this article sets 2 as the maximized evaluable load supply adequacy limit and should stop evaluating if current index has already exceeded the limit. According to the limit setting of load supply adequacy index, this article adopts dichotomy method to determine load growth of next round.

In order to reflect the relativity of load growth among bus load in zone level and system level load supply adequacy evaluation, the growth of load should be allocated to every bus load by rational proportion, which assures evaluation result could reflect the load supply capability 
in future operation. General growth modes of bus load are listed as follows.

1) Proportional growth

All bus loads grow proportionally. This mode is suitable when the bus load is same in type and has same variety rule, or has no scrutiny load model.

2) Proportional growth by load group

Separating bus load into several group by load type, bus loads in same group apply the same growth ratio, and every group could have different growth ratio. This mode is suitable when every type load has different variety rule or integration of intermittent energy generation centrally.

3) Proportional growth by load type

As for zone or system which has scrutiny load model for every bus load, the bus load could be divided into group logically by load type. Then, appropriate growth ratio is selected for every load type to simulate load variation of zone or system.

For multi-period evaluation, the same load growth mode applies for every period generally.

\subsection{Zone Level Load Supply Adequacy Evaluation}

For zone level short term load supply adequacy evaluation in every round, the total load growth is allocated to every bus according to selected load growth mode and load of other zone remaining unchanged. Then optimize unit commitment and output with continuous security constrained generation optimization based evaluation method to satisfy all the constraints. If feasible generation schedule is achieved, then increase load and move to next round evaluation. Otherwise terminate evaluation process and select load supply adequacy index of last round as result for evaluated zone. As for same load growth ratio in every period, the load supply adequacy of every period is same.

Repeating above evaluation process for every zone, the load supply adequacy index of every zone could be achieved.

\subsection{System Level Load Supply Adequacy Evalu- ation}

For system level short term load supply adequacy evaluation in every round, the system load growth is allocated to every bus according to selected load growth mode and keeping tie line exchange fixed. Then optimize unit commitment and output with continuous security constrained generation optimization based evaluation method to satisfy all the constraints. If feasible generation schedule is achieved, then increase load and move to next round evaluation. Otherwise terminate evaluation process and select load supply adequacy index of last round as result for evaluated system. As for same load growth ratio in every period, the load supply adequacy of every period is same.

\section{Design of Online Multi-level Short Term Load Supply Adequacy Evaluation System}

The functional infrastructure of online short term load adequacy evaluation system is demonstrated as Figure 2, the main functions are data management, bus level evaluation, zone level evaluation, system level evaluation, sensitivity factor based evaluation, continuous security constrained generation optimization based evaluation and parameter management. The continuous generation optimization based evaluation module is composed of generation optimization and security analysis.

Data management module serves for model and operation data input, which include network model, outage schedule of generation and transmission devices, transmission capability, system load forecast, bus load forecast, from operation schedule application to form base case for evaluation. Bus level evaluation module implements evaluation data preparation, calling of sensitivity factor based evaluation service, continuous security constrained generation optimization based evaluation service and demonstration of evaluation result. Zone level

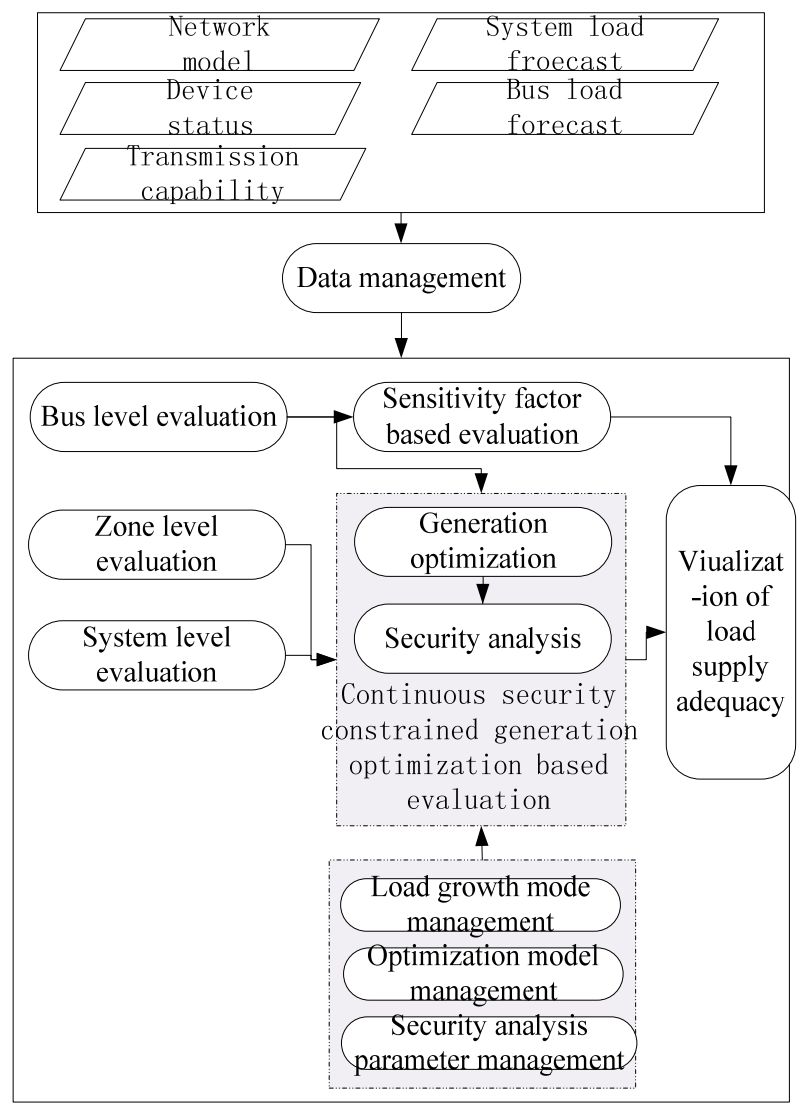

Figure 2. Functional infrastructure of online multi-level short term load supply adequacy evaluation system. 
evaluation module implements load growth management of zones, calling of continuous generation optimization based evaluation service and demonstration of evaluation result of every zone. System level evaluation module implements load growth management of system, calling of continuous generation optimization based evaluation service and demonstration of evaluation result of system. Sensitivity factor based evaluation module provides sensitivity calculation service and sensitivity factor based load supply adequacy evaluation service. Continuous security constrained generation optimization module provides multi-objective, security constrained unit commitment and economic dispatch service, provides single period and multi-periods coupled load supply adequacy evaluation service and calling of base case power flow analysis, contingency analysis and sensitivity calculation service provided by security analysis module automatically. Parameter management module implements load growth mode management, objective model and constraint model management for generation optimization, and parameter management for security analysis. Visualization of load supply adequacy module demonstrates load supply adequacy distribution of transmission network by contour and three dimensional bar methods.

Continuous security constrained generation optimization based evaluation method not only could apply for single period and multi-periods zone level evaluation and system level evaluation for large scale transmission network, but also could apply for bus level evaluation for small scale transmission network. As for enormous calculation of bus level evaluation, only sensitivity factor based evaluation method could be use for online evaluation in engineering for large scale transmission network.

For example of provincial power grid with 700 calculating node, the evaluation time of 96 periods system level evaluation is about 8 minutes, and the evaluation time of 96 periods zone level is about 5 minutes by one ThinkPad computer equipped with $256 \mathrm{G}$ hard disk and $6 \mathrm{G}$ memory. The performance could meet the requirement of online application in engineer.

\section{Conclusions}

This article presents multi-level, including bus level, zone level and system level, load supply adequacy evaluation method, which is able to evaluate load supply adequacy of transmission network at any operation status and provide reliable technical support for coping with random fluctuation caused by intermittent energy genera- tion. After analysis of sensitivity factor based evaluation method, it presents a high performance continuous security constrained generation optimization based evaluation method, which is more feasible in engineering application for zone level and system level load supply adequacy evaluation. And provide the functional design of online multi-level short term load supply adequacy evaluation system, which could be integrated into operation schedule applications. Though the evaluation focuses on load growth mode analysis, same theory could be used in load decreasing analysis so as to cope with the influence of bidirectional fluctuation of large scale intermittent energy generation.

\section{REFERENCES}

[1] L. R. Xie, Z. L. Yang, C. Zhang and J. F. Huan, "Security Evaluation of Maintenance Schedule Based on Load Supply Adequacy and Generation Loss," Power System Automation Conference, 2010.

[2] Z. Q. Ge, D. X. Wang and M. H. Ge, "Security Checking System for Daily Generation Scheduling of East China Power Grid and Its Expansion," Automation of Electric Power Systems, Vol. 32, No. 10, 2008, pp. 45-48.

[3] Z. X. Chen, K. Xie and J. Zhang, "Optimal Model and Algorithm for Day-ahead Generation Scheduling of Transmission Grid Security Constrained Convention Dispatch," Automation of Electric Power Systems, 2009, Vol. 33, No. 1, pp. 10-13.

[4] J. Geng Jian, F. Xu Fan and J. G. YAO, "Performance Analysis of Mixed-integer Programming Based Algorithm for Security Constrained Unit Commitment," Automation of Electric Power Systems, Vol. 33, No. 21, 2009, pp. 24-27,

[5] N. Zhang, H. K. Chen, X. M. Luo, et al., "Model and Algorithm of Energy-conservation Based Generation Dispatching for Guangdong Power Grid," Power system Technology, Vol. 32, No. 24, 2008, pp. 11-15.

[6] X. L. Li, J. Y. Zhou and Y. Erkeng, "Linear Mixed Integer Programming Algorithm for Unit Commitment Based on Dynamic Search," Automation of Electric Power Systems, Vol. 32, No. 21, 2008, pp. 18-21.

[7] G. W. Chang, M. Aganagic, J. G. Waight, J. Medina, T. Burton, S. Reeves and M. Christoforidis, "Experiences with Mixed Integer Linear Programming Based Approaches on Short-term Hydro Scheduling," IEEE Transactions Power Syst, Vol. 16, No. 4, 2001, pp. 743-749.

[8] Z. L. Yang, G. Q. Tang and L. L. Li, "Application of Relaxed Constraints Model on Generation Schedule Optimization," Automation of Electric Power Systems, Vol. 34, No. 14, 2010, pp. 53-57, 